\title{
Vitiligo en niños: enfoque clínico
}

Vitiligo in children: clinical approach

\section{Claudia Juliana Díaz', Jairo Hernán Rodríguez ${ }^{2}$}

1. Médica dermatóloga; docente, Universidad del Valle, Hospital Universitario del Valle Evaristo García, E.S.E., Santiago de Cali, Colombia

2. Médico, residente de tercer año de Dermatología y Cirugía Dermatológica, Universidad del Valle, Hospital Universitario del Valle Evaristo García, E.S.E., Santiago de Cali, Colombia

\section{RESUMEN}

El vitíligo es una enfermedad despigmentante que afecta entre el 1 y el $2 \%$ de la población mundial y cerca de la mitad de los casos se inician en la infancia. Los estudios de prevalencia en diferentes poblaciones de niños, adolescentes y adultos, muestran porcentajes similares en diferentes países del mundo.

Hasta el momento, existen pocos estudios de seguimiento a largo plazo sobre el tratamiento en niños; la mayoría de las publicaciones se enfocan en conceptos preexistentes que son básicos en el estudio y la aproximación terapéutica del paciente pediátrico con vitíligo.

En esta revisión, se resumen la clasificación, las generalidades, las enfermedades asociadas al vitíligo segmentario y no segmentario, las alteraciones psicológicas que presentan los niños afectados por esta condición y sus padres, y el tratamiento, con énfasis en la 'evidencia' clínica sobre los medicamentos más usados.

PAlabRAS Clave: vitiligo, niños, tratamiento

\section{SUMMARY}

Vitiligo is a depigmenting disease that affects between 1 and $2 \%$ of the world population and about half of the cases begin in childhood. The prevalence studies in different populations in children, adolescents and adults show similar percentages in different countries of the world.

So far, there are few long-term follow-up studies regarding treatment in children. Most of the publications focus on preexisting concepts that are basic in the study and in the therapeutic approach of the pediatric patient with vitiligo. In this review, we summarize its classification, generalities, diseases associated with segmental and non-segmental vitiligo, the psychological alterations presented by children affected by this condition and their parents, and the treatment emphasizing the clinical evidence of the most commonly used medications.

KEY WORDS: vitiligo, children, treatment

\author{
Correspondencia: \\ Claudia Juliana Díaz \\ Email: \\ claudia.diaz@correounivalle. \\ edu.co \\ Recibido: $22 / 10 / 17$ \\ Aceptado: 06/05/18

\section{Conflictos de interés:} \\ No se reportan conflictos de \\ interés. \\ Financiación: \\ Ninguna.
}




\section{INTRODUCCIÓN}

El vitíligo es una enfermedad despigmentante poligénica y multifactorial, de presentación variable y común en todas las razas, que afecta cerca del $2 \%$ de la población mundial ${ }^{(1)}$. En el 37 \% de los pacientes con vitíligo, la enfermedad se manifiesta antes de los 12 años de edad ${ }^{(2,3)}$.

Se caracteriza por máculas despigmentadas de bordes bien definidos que comprometen diferentes áreas corporales. En algunos casos, el vitiligo puede presentar un borde inflamatorio y ocasionar prurito. El diagnóstico es clínico y el examen bajo la luz de Wood es de gran ayuda pues resalta la despigmentación, y permite diferenciarlo de otras enfermedades que se presentan con hipopigmentación.

En los niños, el vitíligo generalizado es la forma más frecuente; sin embargo, en comparación con los adultos, el segmentario se presenta con mayor frecuencia.

El vitiligo segmentario se caracteriza por un inicio temprano (menor edad promedio de inicio que el vitíligo no segmentario), cuya presentación clínica es focal, en las mucosas, unisegmentario, bisegmentario o plurisegmentario. En el $90 \%$ de los casos, compromete un solo segmento y, en orden de frecuencia, se localiza principalmente en la cabeza y el cuello, seguidos por el tronco y las extremidades. Inicialmente, su progresión es rápida y luego se estabiliza, acompañado de leucotriquia temprana en la lesión y en su periferia. En más del $50 \%$ de los pacientes, progresa en los dos primeros años, se asocia ocasionalmente con enfermedades autoinmunitarias, con el fenómeno de Köebner, y antecedentes familiares de vitíligo. Hay poca mejoría con el tratamiento médico y, cuando no responde a la combinación de medicamentos tópicos convencionales, la opción es el tratamiento quirúrgico ${ }^{(4,5)}$.

Se han propuesto varias hipótesis sobre su patogenia, como la autoinmunitaria, la autocitotóxica y la neural; no obstante, la etiología principal es de carácter autoinmunitario, relacionada con mutaciones genéticas específicas y con un gran polimorfismo. Se cree que la respuesta inflamatoria del vitiligo está mediada por genes como FOXD 3 , ACE, APE, GSTP1, TLR, SOD y $C T L A-4$, entre otros, relacionados con la destrucción de los melanocitos, con mediación de la inmunidad celular, la inmunidad humoral por anticuerpos y la acción de las citocinas.

Estos planteamientos se basan, principalmente, en la asociación clínica del vitiligo con varios trastornos autoinmunitarios que comparten genes como el HLA$D R B 1 /-D Q A 1$, el PTPN22, el BACH 2 y el $C T L A-4$, entre los más importantes, y que se relacionan con otras en- fermedades como la tiroiditis ${ }^{(6-9)}$, la alopecia areata, la enfermedad intestinal inflamatoria, la esclerosis múltiple, la miastenia gravis, la diabetes de tipo 1, el lupus eritematoso sistémico, la hepatitis autoinmunitaria, el halo nevus y la pérdida de la audición neurosensorial. Esta última ha sido estudiada en varios pacientes con vitiligo, debido a la reducción del número de melanocitos contenidos en el laberinto membranoso del oído interno $^{(10-15)}$.

Otras teorías, como la del estrés oxidativo (oxidative stress) y la producción de especies reactivas de oxígeno, sostienen que estas inician una cascada de señalizaciones que activarían la producción de proteínas de choque térmico, perpetuando la reacción inflamatoria de las citocinas ${ }^{(16,17)}$. Asimismo, la teoría neural sigue considerándose por la presencia de diferentes tipos de vitíligo segmentario, y el compromiso en áreas denervadas y en modelos animales.

Recientemente, las citocinas secretadas por las células Th17 han adquirido importancia por su relevante papel en el mantenimiento y la propagación del vitíligo, ya que aumentan de acuerdo con la extensión de la enfermedad. Se ha encontrado una significativa elevación de las interleucinas (IL) IL-2 e IL-6 en el vitíligo localizado, y de IL-17 e IL-22, en el generalizado. El TNFa también aumenta en proporción con la actividad de la enfermedad, por lo tanto, puede usarse como biomarcador para identificar una enfermedad agresiva. Además, se ha demostrado que la IL-22 está involucrada en la inducción y en la producción de especies reactivas de oxígeno (ROS), promoviendo la activación del inflamosoma (NLRP3) y las caspasas, con la consecuente destrucción del queratinocito ${ }^{(17,18)}$.

Debido a su compleja patogenia, existen varias opciones terapéuticas disponibles, cuya escogencia depende básicamente del tipo de vitiligo y de la extensión de las lesiones. No obstante, hay otros factores muy importantes que influyen en las decisiones, como la edad del paciente, la progresión o estabilidad del vitíligo, las comorbilidades y las alteraciones psicológicas del paciente. Hasta el momento, existen pocos estudios de seguimiento a largo plazo en niños, y se están estudiando nuevos tratamientos ${ }^{(19,20)}$.

\section{CLASIFICACIÓN}

Según sus manifestaciones clínicas, el vitiligo se clasifica en segmentario, no segmentario, mixto y no clasificado; hay otros tipos, como el punctata (hoy llamado leucodermia punctata), el cual se incluye preferentemente en el grupo de los no segmentarios ${ }^{(21)}$. 
En general, el vitíligo de las mucosas compromete la mucosa oral, la genital o ambas; el acrofacial se caracteriza por máculas en áreas distales de los miembros, genitales, cara y zonas periorificiales, y puede progresar a la forma generalizada o universal; el generalizado afecta varias partes corporales con tendencia a ser simétrico; el universal produce despigmentación corporal completa o casi completa; el focal consiste en una lesión despigmentada, focal, pequeña y aislada, que generalmente no evoluciona a vitíligo no segmentario, el segmentario se refiere a lesiones maculares en uno, dos o más segmentos de la piel; y el mixto consiste en la coexistencia del no segmentario y el segmentario.

\section{ENFERMEDADES ASOCIADAS}

El vitíligo segmentario es más frecuente en niños que en adultos y, también, en niñas que en niños sin diferencias significativas. Es considerado de diferente etiología, su inicio es rápido y tiende a estabilizarse con el tiempo, sin embargo, se puede reactivar en la vida adulta $^{(22)}$.

El vitiligo no segmentario es el más común en los niños. Es de distribución bilateral, compromete las mucosas, región, acrofacial o generalizado. El vitiligo segmentario y el no segmentario se relacionan con diversas condiciones, como enfermedades autoinmunitarias, deficiencias vitamínicas y alteraciones psicológicas. La asociación entre el vitíligo y las enfermedades autoinmunitarias no ha sido plenamente explicada, y es menor en el caso del segmentario. El estudio del genoma ha aportado importantes conocimientos para dilucidar el papel compartido del vitiligo con dichas condiciones patológicas. También, se ha asociado con disfunción de la tiroides, especialmente hipotiroidismo (5,4\%), artritis reumatoidea $(1,1 \%)$, psoriasis $(1,1 \%)$, alopecia areata (o,8 \%), enfermedad celiaca, enfermedad de Addison y pénfigo vulgar. La relación entre vitíligo y enfermedad tiroidea es la más reportada en múltiples estudios, y se considera que los niños y los adolescentes afectados deben estudiarse regularmente para descartar este compromiso, principalmente cuando la presentación es no segmentaria $(23,24)$. Debe tenerse en cuenta que la frecuencia de las enfermedades autoinmunitarias pueden relacionarse con los antecedentes familiares y con la raza ${ }^{(23,24)}$.

El vitíligo segmentario se ha asociado con dermatitis atópica, alopecia areata, liquen escleroso extragenital y halo nevus, este último sirve como marcador de progresión a vitíligo mixto ${ }^{(25-27)}$. La disfunción coclear también se ha evaluado en niños y adultos, y se ha en- contrado que, independientemente del tipo de vitíligo, existe alteración neurosensorial, lo que sugiere coincidencias en su patogenia ${ }^{(28)}$.

En varias enfermedades autoinmunitarias se ha estudiado el papel de la vitamina D. Se ha informado disminución de la concentración de $25-\mathrm{OH}$ vitamina $\mathrm{D}$ en niños y en adultos con vitiligo, y se ha sugerido que esto podría indicar la presencia o la tendencia hacia la autoinmunidad en niños, en quienes podría ser más frecuente el desarrollo de enfermedad tiroidea, diabetes mellitus y anticoagulante lúpico. Se debe sospechar déficit de vitamina D cuando se presenten lesiones 'en confeti' en pacientes que presentan hipopigmentación o hiperpigmentación que sugieran déficit nutricional ${ }^{(29)}$.

Según lo anteriormente expuesto, algunos autores han recomendado una evaluación anual de la función tiroidea o de la concentración de vitamina $\mathrm{D}$, según el caso, en pacientes con vitíligo no segmentario o con antecedentes de enfermedades familiares autoinmunitarias. Otros exámenes de laboratorio, como hemograma, anticuerpos antinucleares (ANA), glucemia, factor reumatoideo y niveles de ácido fólico, de vitamina $\mathrm{B}_{6} \mathrm{y}$ de $\mathrm{B}_{12}$, deben solicitarse según las manifestaciones clínicas de cada paciente ${ }^{(30)}$.

\section{TRASTORNOS PSICOLÓGICOS ASOCIADOS CON EL VITILIGO}

El vitiligo puede llegar a ser psicológicamente devastador y un estigma, especialmente en las personas de piel oscura. Mediante encuestas de calidad de vida y autopercepción, se ha demostrado que los pacientes con vitiligo y sus familiares presentan alteraciones psicológicas ${ }^{(31)}$. Los afectados suelen padecer con mayor frecuencia depresión y disminución de la calidad de vida; los adolescentes con compromiso extenso en la cara o afectación genital, presentan alteraciones escolares, falla escolar, baja autoestima y dificultad en su desarrollo sexual ${ }^{(32)}$.

Los médicos no dermatólogos y los dermatólogos deben estar alerta y saber reconocer el gran impacto de la enfermedad, y prestar la debida ayuda, con remisión a consulta de psicología o psiquiatría, para abordar los factores objetivos y subjetivos de la enfermedad ${ }^{33,34)}$. 


\section{TRATAMIENTO}

Se han empleado múltiples tratamientos con el fin de detener la progresión y obtener repigmentación del vitiligo; cabe resaltar que las terapias combinadas son superiores que la monoterapia ${ }^{(35,36)}$.

\section{CORTICOSTEROIDES TÓPICOS}

Los corticosteroides tópicos se consideran el tratamiento de primera línea con los inhibidores de calcineurina, tanto en niños como en adultos, y son seguros y eficaces a corto plazo. Su uso prolongado produce efectos secundarios, como atrofia cutánea, telangiectasias, hipertricosis, erupciones acneiformes y estrías. En la mayoría de los estudios en niños se han empleado el propionato de clobetazol y la mometasona, comparándolos con inhibidores de la calcineurina (pimecrolimus y tacrolimus). En un estudio de 50 pacientes con vitiligo infantil, se comparó el pimecrolimus con la mometasona en dos grupos, cada uno de 20 niños que completaron el estudio; durante tres meses, un grupo se trató con mometasona en crema al o,1 \% una vez al día, y el otro, con pimecrolimus en crema al $1 \%$ dos veces al día. El tamaño de las lesiones disminuyó en forma estadísticamente significativa con ambos fármacos. Al final del tratamiento, la tasa media de repigmentación fue del $65 \%$ con la mometasona y de $42 \%$ con el pimecrolimus. Se concluyó que el furoato de mometasona puede aplicarse en cualquier parte del cuerpo ${ }^{(37,38)}$.

En niños con vitiligo extrafacial, un corticosteroide tópico potente no debe usarse por un período mayor de tres meses. Los de menor potencia deben ser aplicados una vez diaria durante 15 días al mes, durante seis meses.

Por otra parte, se ha estudiado el efecto de combinar corticosteroides con tretinoína ${ }^{\left({ }^{39}\right)}$. En una serie de 50 pacientes, que incluyó 12 menores de 20 años, la aplicación diaria de clobetazol tópico combinado con tretinoína durante seis meses, aumentó la velocidad de la repigmentación de las lesiones faciales y corporales, con buena reacción terapéutica en $55 \%$ de los casos ${ }^{(40)}$.

\section{INHIBIDORES DE CALCINEURINA}

Los inhibidores de calcineurina son particularmente efectivos en cabeza y cuello, sin que produzcan efectos adversos importantes, en comparación con los corticosteroides tópicos; los estudios comparativos entre el tacrolimus al 0,1 \% y el clobetazol, ofrecen simi- lares resultados en el vitiligo facial y el extrafacial. En adultos se recomiendan dos aplicaciones diarias en combinación con exposición moderada al sol, inicialmente durante seis meses. En niños con vitiligo, aún no se ha evaluado su uso a largo plazo; sin embargo, los resultados son tranquilizadores en condiciones como la dermatitis atópica (figura 1) (41-44).

Un metaanálisis sugiere que la adición de inhibidores tópicos de calcineurina y análogos tópicos de vitamina D3 con fototerapia UVB-NB, puede producir resultados significativamente superiores que la fototerapia sola, en cara y cuello ${ }^{(45)}$.

\section{ANÁLOGOS DE LA VITAMINA D (CALCIPOTRIOL Y CALCITRIOL)}

Se han obtenido resultados variables de repigmentación con compuestos análogos de la vitamina D en estudios prospectivos no controlados. No se recomienda el calcipotriol tópico como monoterapia; estos medicamentos deben usarse combinados con fototerapia UVB-NB o con corticosteroides tópicos, aunque no hay 'evidencia' suficiente para recomendar su uso en niños ${ }^{(46-48)}$.

\section{CORTICOSTEROIDES SISTÉMICOS}

Los corticosteroides sistémicos administrados en pulsos orales detienen la progresión de la enfermedad e inducen repigmentación cuando se utilizan durante el inicio de un vitiligo rápidamente progresivo. En niños menores de 15 años se administró metilprednisolona oral y se hizo seguimiento de un año; las recaídas fueron más frecuentes en los menores de 10 años en comparación con los de mayor edad ${ }^{(49,50)}$.

\section{FOTOTERAPIA CON RADIACIÓN ULTRAVIOLETA}

La fototerapia UVB-NB es la modalidad de tratamiento más usada en el vitiligo generalizado y se considera que produce menos efectos adversos que el tratamiento de psoralenos más UVA (PUVA) e, incluso, muestra una eficacia superior. Se asocia con efectos secundarios como eritema, picazón y ardor leve o dolor que, en la mayoría de los casos, son bien tolerados y desaparecen espontáneamente unas pocas horas después del tratamiento. 
Se han llevado a cabo varios estudios en los cuales se incluyen niños tratados con esta terapia, principalmente con luz ultravioleta de banda estrecha (UVB$\mathrm{NB})$. Esta se recomienda, especialmente, cuando las lesiones se extienden en más del 15 al $20 \%$ del área corporal o en el vitíligo generalizado con gran impacto en la calidad de vida.

Se aconsejan dos a tres sesiones semanales hasta por seis meses, aunque, hasta el momento, no existe consenso sobre la duración óptima del tratamiento ni sobre el número de sesiones; se ha sugerido que podrían ser hasta 200 sesiones.

Muchos médicos tratantes tienden a suspender la irradiación si no se obtiene repigmentación durante los tres primeros meses de tratamiento o si, después de seis meses, la respuesta terapéutica no es satisfactoria, con menos de $25 \%$ de repigmentación. Tampoco hay consenso sobre la edad de inicio, pero se sugiere que sea después de los 12 años de edad; igualmente, es conveniente explicar a los padres los efectos adversos del tratamiento, como prurito, descamación, eritema, dolor, ampollas y fototoxicidad.

En niños asiáticos con vitiligo, se estudiaron diferentes modalidades terapéuticas y se encontraron mayores tasas de mejoría con la UVB-NB, seguida por la combinación de UVB más UVA-1 ${ }^{\left({ }^{51-53)}\right.}$.

Actualmente, el tratamiento más innovador es la fototerapia objetivo o microfoco UVB, que permite al operador tratar solo las áreas hipopigmentadas, respetando la piel no afectada, usando los mismos mecanismos de la fototerapia clásica mediante un generador de luz fría ${ }^{(54)}$.

El uso de la UVA-1 de 300 a $400 \mathrm{~nm}$ es relativamente reciente en dermatología, y sus efectos a largo plazo en el vitiligo no se han investigado. Se suele usar tres a cinco sesiones semanales; se inicia con 20 a $30 \mathrm{~J} / \mathrm{cm} 2$ y se aumentan progresivamente. Además, existe la UVA-1 focalizada de $355 \mathrm{~nm}$, cuyos efectos secundarios agudos son raros, como eritema y prurito, y otros a largo plazo que aún no se han establecido ${ }^{(54)}$.
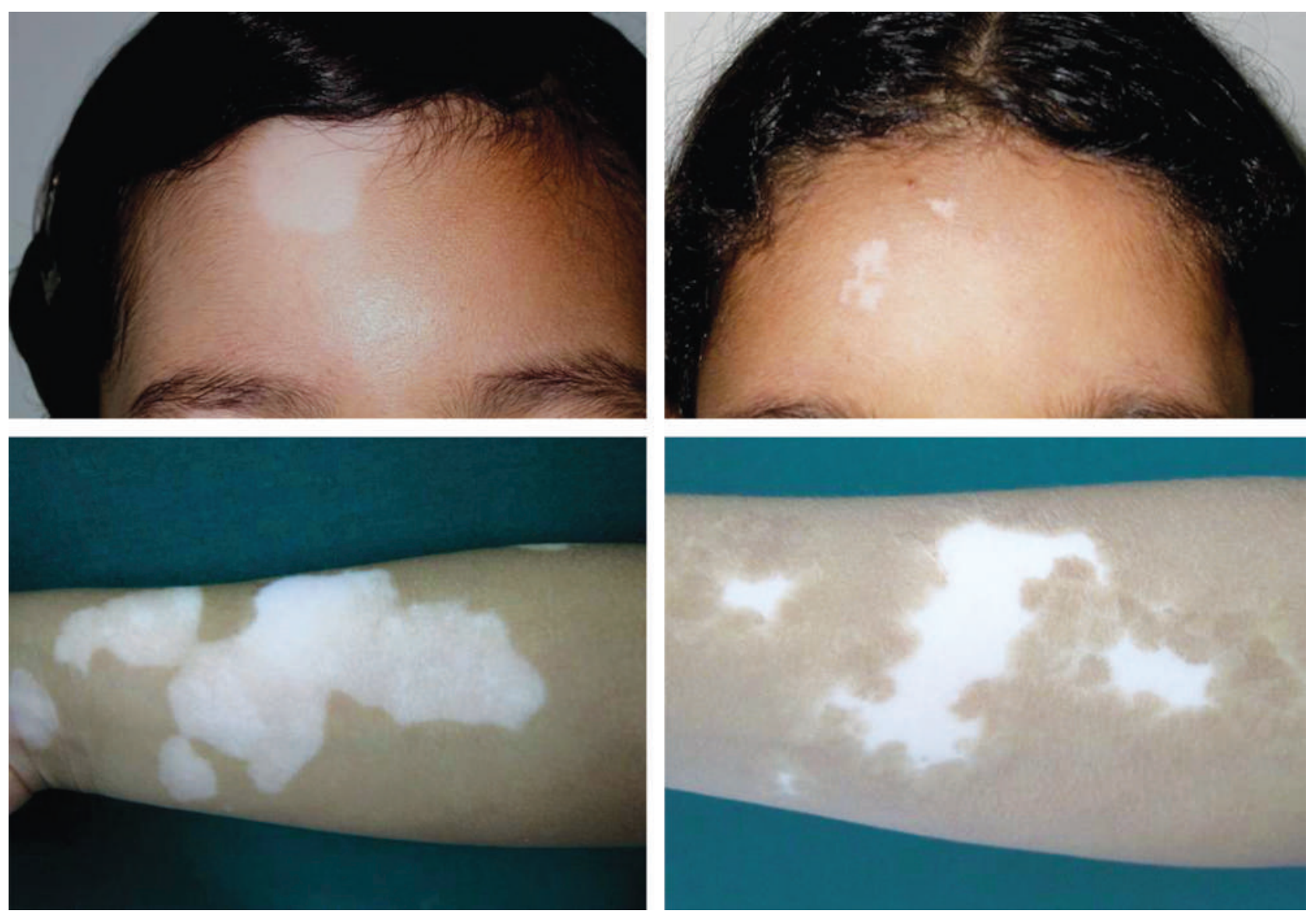

Figura 1. Vitiligo segmentario en la frente y el antebrazo, tratamiento por nueve meses con tacrolimus al o,1 \%, dos veces al día en diferentes pacientes 


\section{LÁSER DE EXCÍMEROS}

El láser de excímeros (excimer laser) de $308 \mathrm{~nm}$, de cloruro de xenón, con un tamaño de punto variable (valor medio: 15 a $25 \mathrm{~mm}$ ) se ha usado en lesiones localizadas, pequeñas y de reciente aparición, pero es más efectivo cuando se combina con inhibidores de la calcineurina y corticosteroides tópicos ${ }^{(55)}$. El esquema terapéutico varía según las características del paciente y de la enfermedad.

En un estudio de 48 pacientes, que incluyó niños afectados con vitíligo localizado, generalizado, acrofacial y de tipo segmentario, se evaluó la eficacia de la luz monocromática excimer de $308 \mathrm{~nm}$ (Monochromatic Excimer Light, MEL) como monoterapia, en comparación con su combinación con kelina al 4 \%; las lesiones con la mejor tasa de mejoría fueron las de inicio reciente, con vitiligo localizado en la cara, el cuello y las manos, tratadas con dicha combinación ${ }^{(56,57)}$.

La tasa y la velocidad de la repigmentación con este láser varían según la localización y la duración de la enfermedad. Las lesiones en la cara y el cuello reaccionan muy bien, mientras que las de las extremidades muestran una mejoría más lenta ${ }^{(58)}$.
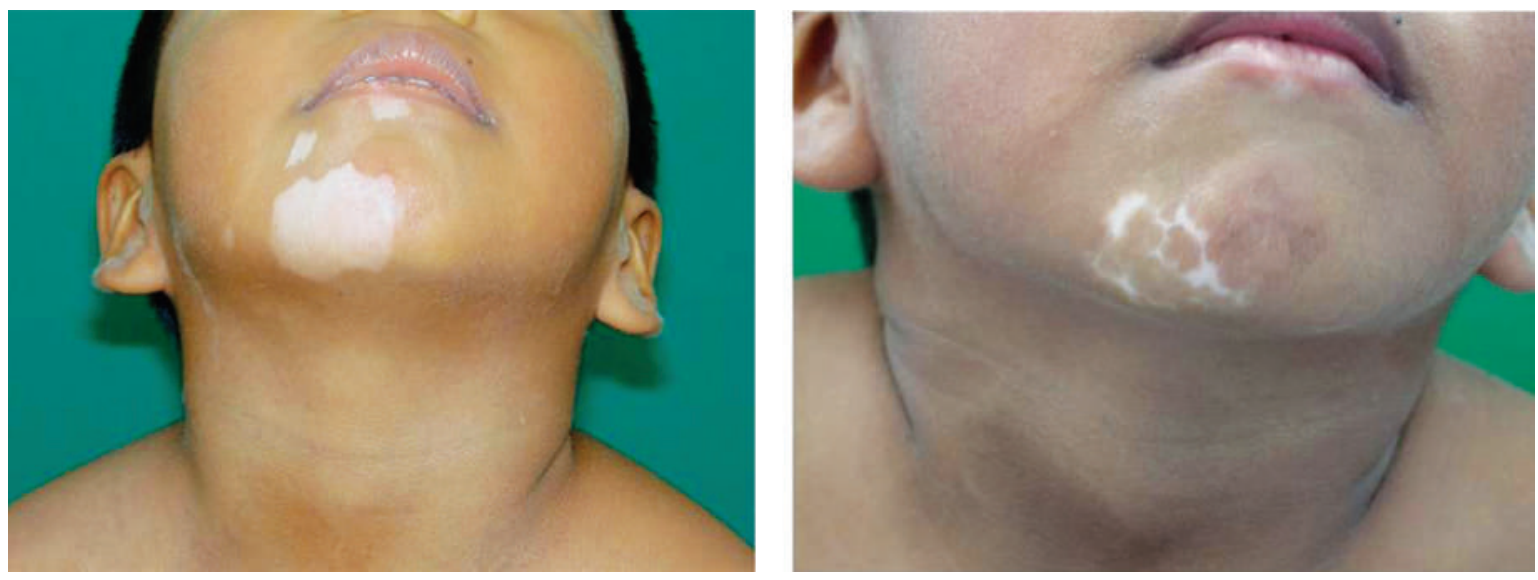

Figura 2. Vitiligo segmentario después de cinco meses de tratamiento con kelina al $3 \%$ tres veces por semana

\section{FOTOQUIMIOTERAPIA}

En algunos países, se ha usado la combinación de psoralenos y luz ultravioleta A (PUVA tópica) para el vitíligo localizado en niños mayores de dos años, cuando no ha habido mejoría después de seis meses de tratamiento con corticosteroides tópicos; sin embargo, se consideran tratamientos de segunda línea. Consiste en la aplicación tópica, una a dos veces por semana, de crema o ungüento (o,01-0,1 \%) de 8-metopxisoraleno (8-MOP) 30 minutos antes de la exposición a la UVA en dosis de 0,12 a $0,25 \mathrm{~J} / \mathrm{cm}^{2}{ }^{(59)}$. Los efectos secundarios más comunes con la terapia PUVA son eritema fototóxico, prurito, foliculitis y pigmentación, y rara vez, dermatitis de contacto ${ }^{(60-62)}$.

\section{KELINA}

La kelina es un furocromo derivada de la planta bizvitiligo, en algunos países. Su combinación con radiación ultravioleta A recibe el nombre de KUVA (kelina más UVA). Se usa en crema o gel al 3 a $5 \%$, aplicada a diario o tres veces por semana 30 minutos antes de la exposición solar, con lo cual se obtienen resultados beneficiosos. Se ha reportado su eficacia en asociación con rayos ultravioleta $\mathrm{A}$, contribuyendo en la activación de la mitogénesis y migración de los melanocitos; sin embargo, faltan estudios aleatorizados y controlados con otros tipos de tratamiento, para evaluar su utilidad a largo plazo en niños (figura 2) ${ }^{(63,64)}$. naga (Ammi visnaga), usada en adultos y en niños con 


\section{FENILALANINA}

La fenilalanina es segura en niños y no se han reportados efectos secundarios. En un estudio no controlado de 13 pacientes pediátricos, el tratamiento con $100 \mathrm{mg} /$ $\mathrm{kg}$ de fenilalanina con fototerapia UVA, dio como resultado la repigmentación completa en tres pacientes, y de 50 a $90 \%$ de mejoría en seis pacientes. La repigmentación se produjo con mayor facilidad en la cara y las extremidades.

En un estudio retrospectivo no controlado llevado a cabo entre 1990 y 1995, se usó fenilalanina tópica al 10 $\%$ en gel y por vía oral en dosis diarias de $500100 \mathrm{mg} /$ kg, para tratar el vitíligo en 171 pacientes, incluidos 52 niños menores de 15 años; el resultado fue mejoría total en el 83,1 \% de los casos y, en la cara, en el 90,3 \% ${ }^{(65,66)}$.

\section{TRATAMIENTO QUIRÚRGICO}

Las intervenciones quirúrgicas consisten en injertos de ampollas, trasplante de células cultivadas, trasplante de células no cultivadas, e injertos con sacabocados o miniinjertos de piel.

La cirugía se reserva para los pacientes que no han mejorado con otras formas de tratamiento y puede ser eficaz para áreas pequeñas en niños con enfermedad estable. No hay consenso sobre la edad para utilizar el tratamiento quirúrgico y podría plantearse en niños mayores de 10 años.

Los injertos de ampollas por succión, combinado o con tratamiento tópico, ofrecen los mejores resultados. Hay estudios recientes con un número considerable de pacientes, que apoyan el tratamiento quirúrgico con cultivos de melanocitos en adolescentes, pero se requieren laboratorios sofisticados y un entrenamiento especial. Hay informes sobre el tratamiento con miniinjertos en niños; son una opción económica y sencilla que, realizada por dermatólogos entrenados en la técnica, brinda resultados satisfactorios sin efectos adversos. De todas maneras, debe considerarse el tratamiento según la edad del paciente y establecer las expectativas en conjunto con los padres, explicando los efectos secundarios que podrían generarse con el tratamiento (figura 3) ${ }^{(67-71)}$.

En conclusión, el vitíligo debe evaluarse como una enfermedad multifactorial, descartando enfermedades asociadas y haciendo una intervención psicológica familiar temprana, según sea el caso. Las posibilidades de tratamiento en niños con vitíligo son variadas, pero el tratamiento combinado es la mejor opción. Hay nuevos tratamientos en evaluación, como la UVA-1 y la terapia focal con UVB, y faltan estudios a largo plazo. Teniendo en cuenta la fisiopatología, otros tratamientos serán objeto de estudio a largo plazo, como las dosis bajas de citocinas antiinflamatorias, los factores de crecimiento, los neuropéptidos como el FGF, y las interleucinas IL-4, IL-10 e IL-1, que ya se mencionan en la literatura y que podrían ser útiles en un futuro, en combinación con terapias convencionales, para restaurar los queratinocitos alterados y su relación con el melanocito.
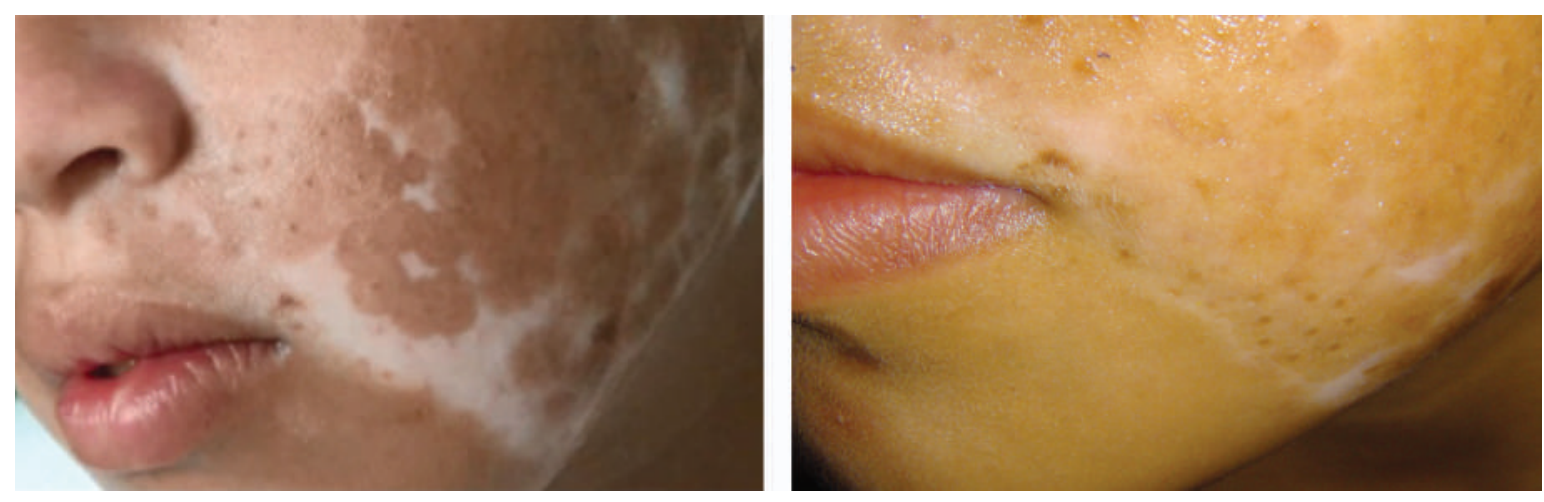

Figura 3. Evolución de vitiligo estable con tratamiento tópico con tacrolimus al o,1% dos veces al día y múltiples intervenciones con miniinjertos de $1 \mathrm{~mm}$ : repigmentación del área afectada 


\section{REFERENCIAS}

1. Kruger C, Schallreuter KU. A review of the worldwide prevalence of vitiligo in children/adolescents and adults. Int J Dermatol. 2012;51:1206-12.

2. Sehgal VN, Srivastava G. Vitiligo: Compendium of clinico-epidemiological features. Indian J Dermatol Venereol Leprol. 2007;73:149-56.

3. Ezzedine K, Eleftheriadou V, Whitton M, van Geel N. Vitiligo. Lancet. 2015;386:74-84.

4. Taieb A, Morice-Picard F, Jouary T, Ezzedine K, et al. Segmental vitiligo as the possible expression of cutaneous somatic mosaicism: Implications for common non-segmental vitiligo. Pigment Cell Melanoma Res. 2008;21:646-52.

5. Ezzedine K, Le Thuaut A, Jouary T, Ballanger F, Taieb A, Bastuji-Garin S. Latent class analysis of a series of 717 patients with vitiligo allows the identification of two clinical subtypes. Pigment Cell Melanoma Res. 2014;27:134-9.

6. Xianfeng C, Yuegen J, Zhiyu Y, Yan Y, Xuesi Z, Fenglai W, et al. Pediatric patients with vitiligo in eastern China: Abnormalities in 145 cases based on thyroid function tests and Immunological findings. Med Sci Monit. 2015;21:3216-21.

7. Kakourou T, Kanaka-Gantenbein C, Papadopoulou A, Kaloumenou E, Chrousos GP. Increased prevalence of chronic autoimmune (Hashimoto's) thyroiditis in children and adolescents with vitiligo. J Am Acad Dermatol. 2005;53:220-3.

8. Baldini E, Odorisio T, Sorrenti S, Catania A, Tartaglia F, Carbotta G, et al. Vitiligo and autoimmune thyroid disorders. Front Endocrinol (Lausanne). 2017;27:290.

9. Aslan S, Serarslan G, Teksoz E, Dagli S. Audiological and transient evoked otoacoustic emission findings in patients with vitiligo. Otolaryngol Head Neck Surg. 2010;142:409-14.

10. Chen YT, Chen YJ, Hwang CY, Lin MW, Chen TJ, Chen CC, et al. Comorbidity profiles in association with vitiligo: A nationwide population-based study in Taiwan. J Eur Acad Dermatol Venereol. 2015;29:1362-9.

11. Sharma CK, Sharma M, Prasad K. Involvement of different genes expressions during immunological and inflammatory responses in vitiligo.Crit Rev Eukaryot Gene Expr. 2017;27:277-87.

12. Spritz RA, Andersen GH. Genetics of vitiligo.Dermatol Clin. 2017;35:245-55.

13. Dammak I, Boudaya S, Ben Abdallah F, Turki $\mathrm{H}$, Attia H, Hentati B. Antioxidant enzymes and lipid peroxidation at the tissue level in patients with stable and active vitiligo. Int J Dermatol. 2009;48:476-80.

14. Speeckaert R, van Geel N. Vitiligo: An update on pathophysiology and treatment options. Am J Clin Dermatol. 2017;18:733-44.

15. Rodrigues M, Ezzedine K, Hamzavi I, Pandya AG, Harris JE, Vitiligo Working Group. New discoveries in the pathogenesis and classification of vitiligo. J Am Acad Dermatol. 2017;77:1-13.

16. Elela MA, Hegazy RA, Fawzy MM, Rashed LA, Rasheed $\mathrm{H}$. Interleukin 17, interleukin 22 and FoxP3 expression in tissue and serum of non-segmental vitiligo: A case-controlled study on eighty-four patients. Eur J Dermatol. 2013;23:350-5.

17. Chatterjee S, Eby JM, Al-Khami AA, Soloshchenko M, Kang HK, Kaur N, et al. A quantitative increase in regulatory $\mathrm{T}$ cells controls development of vitiligo. J Invest Dermatol. 2014;134:1285-94

18. Dong J, An X, Zhong H, Wang Y, Shang J, Zhou J. Oncotarget. 2017;8:109161-74.

19. Barygina V, Becatti M, Lotti T, Moretti S, Taddei N, Fiorillo C. Treatment with low-dose cytokines reduces oxidative-mediated injury in perilesional keratinocytes from vitiligo skin. J Dermatol Sci. 2015;79:163-70.

20. Lotti T, Hercogova J, Wollina U, Chokoeva AA, Zarrab Z, Gianfaldoni S, et al. Vitiligo: Successful combination treatment based on oral low dose cytokines and different topical treatments. J Biol Regul Homeost Agents. 2015;29(Suppl.):53-8.

21. Ezzedine K, Lim HW, Suzuki T, Katayama I, Hamzavi I, Lan CC, et al. Revised classification/nomenclature of vitiligo and related issues: The Vitiligo Global Issues Consensus Conference. Pigment Cell Melanoma Res. 2012;25:E1-13.

22. van Geel N, De Lille S, Vandenhaute S, Gauthier Y, Mollet I, Brochez L, et al. Different phenotypes of segmental vitiligo based on a clinical observational study. J Eur Acad Dermatol Venereol. 2011;25:673-8.

23. Silverberg NB. Pediatric vitiligo. Pediatr Clin North Am. 2014;61:347-66.

24. Prcic S, Djuran V, Katanic D, Vlaski J, Gajinov Z. Vitiligo and thyroid dysfunction in children and adolescents. Acta Dermatovenerol Croat. 2011;19:24854 .

25. Weyant GW, Chung CG, Helm KF. Halo nevus: Review of the literature and clinicopathologic findings. Int J Dermatol. 2015;54:433-5.

26. Zhou H, Wu LC, Chen MK, Liao QM, Mao RX, Han JD. Factors associated with development of vitiligo in patients with halo nevus. Chin Med J (Engl). 
2017:20;130:2703-8.

27. Diaz-Angulo S, López-Hoyos M, Muñoz-Cacho P, López-Escobar M, González-López MA. High prevalence of thyroid autoimmunity in patients with alopecia areata and vitiligo: A controlled study. Australas J Dermatol. 2015;56:142-3.

28. Anbar TS, El-Badry MM, McGrath JA, Abdel-Azim ES. Most individuals with either segmental or nonsegmental vitiligo display evidence of bilateral cochlear dysfunction. Br J Dermatol. 2015;172:406-11.

29. Silverberg JI, Silverberg AI, Malka E, Silverberg NB. A pilot study assessing the role of 25 hydroxy vitamin D levels in patients with vitiligo vulgaris. J Am Acad Dermatol. 2010;62:937-41.

30. Silverberg NB. Recent advances in childhood vitiligo. Clin Dermatol. 2014;32:524-30.

31. Amer AA, Mchepange UO, Gao XH, Hong Y, Qi R, $\mathrm{Wu}$ Y, et al. Hidden victims of childhood vitiligo: Impact on parents' mental health and quality of life. Acta Derm Venereol. 2015;95:322-5.

32. Silverberg JI, Silverberg NB. Quality of life impairment in children and adolescents with vitiligo. Pediatr Dermatol. 2014;31:309-18.

33. Sánchez G, Araluce M. Vitiligo: aspectos psicológicos. Rev Hosp Psiquiatr Habana. 1988;29:463-76.

34. Cupertino F, Niemeyer-Corbellini JP, Ramos-ESilva M. Psychosomatic aspects of vitiligo. Clin Dermatol. 2017;35:292-7.

35. van Driessche F, Silverberg N. Current management of pediatric vitiligo. Paediatr Drugs. 2015;17:303-13.

36. Taieb A, Alomar A, Bohm M, Dell'anna ML, De Pase A, Eleftheriadou V, et al. Guidelines for the management of vitiligo: The European Dermatology Forum consensus. Br J Dermatol. 2013;168:519.

37. de Menezes AF, Oliveira de Carvalho F, Barreto RS, de Santana Silva B, Shanmugam S, Gurgel RQ, et al. Pharmacologic treatment of vitiligo in children and adolescents: A systematic review. Pediatr Dermatol. 2017;34:13-24.

38. Kose O, Arca E, Kurumlu Z. Mometasone cream versus pimecrolimus cream for the treatment of childhood localized vitiligo. J Dermatolog Treat. 2010;21:133-9.

39. Kwon HB, Choi Y, Kim HJ, Lee AY. The therapeutic effects of a topical tretinoin and corticosteroid combination for vitiligo: A placebo-controlled, paired-comparison, left-right study. J Drugs Dermatol 2013;12:63-7.

40. Parsad D, Saini R, Juneja A. Combination of clobetasol and tretinoin in vitiligo. Int J Dermatol.
2000;39:639-40.

41. Coskun B, Saral Y, Turgut D. Topical $0.05 \%$ clobetasol propionate versus $1 \%$ pimecrolimus ointment in vitiligo. Eur J Dermatol. 2005;15:88-91.

42. Wong R, Lin AN. Efficacy of topical calcineurin inhibitors in vitiligo. Int J Dermatol. 2013;52:491-6.

43. Silverberg NB, Lin P, Travis L, Farley-Li J, Mancini AJ, Wagner AM, et al. Tacrolimus ointment promotes repigmentation of vitiligo in children: A review of 57 cases. J Am Acad Dermatol. 2004;51:760-6.

44. Grimes PE, Soriano T, Dytoc MT. Topical tacrolimus for repigmentation of vitiligo. J Am Acad Dermatol. 2002;47:789-91.

45. Li R, Qiao M, Wang X, Zhao X, Sun Q . Effect of narrow band ultraviolet $B$ phototherapy as monotherapy or combination therapy for vitiligo: A meta-analysis. Photodermatol Photoimmunol Photomed. 2017;33:22-31.

46. Gargoom AM, Duweb GA, Elzorghany AH, Benghazil M, Bugrein OO. Calcipotriol in the treatment of childhood vitiligo. Int J Clin Pharmacol Res. 2004;24:11-4.

47. Vásquez-López F, López-Escobar M, Pérez-Oliva N. Calcipotriene and vitiligo. Arch Dermatol. 2003;139:1656-7.

48. Parsad D, Saini R, Nagpal R. Calcipotriol in vitiligo: A preliminary study. Pediatr Dermatol. 1999;16:317-20.

49. Majid I, Imran S. Relapse after methylprednisolone oral minipulse therapy in childhood vitiligo: A 12-month follow-up study. Indian J Dermatol. 2013;58:113-6.

50. Majid I, Masood Q, Hassan I, Khan D, Chisti M. Childhood vitiligo: Response to methylprednisolone oral minipulse therapy and topical fluticasone combination. Indian J Dermatol. 2009;54:124-7.

51. Kanwar AJ, Dogra S. Narrow-band UVB for the treatment of generalized vitiligo in children. Clin Exp Dermatol. 2005;30:332-6.

52. Koh MJ, Mok ZR, Chong WS. Phototherapy for the treatment of vitiligo in Asian children. Pediatr Dermatol. 2015;32:192-7.

53. Njoo MD, Bos JD, Westerhof W. Treatment of generalized vitiligo in children with narrow-band (TLo1) UVB radiation therapy. J Am Acad Dermatol. 2000;42:245-53.

54. Gianfaldoni S, Tchernev G, Wollina U, Lotti J, Rovesti M, Satolli F, et al. Vitiligo in children: What's new in treatment? Open Access Maced J Med Sci. 2018;6:221-5.

55. Bae JM, Yoo HJ, Kim H, Lee JH, Kim GM. Combina- 
tion therapy with 308-nm excimer laser, topical tacrolimus, and short-term systemic corticosteroids for segmental vitiligo: A retrospective study of 159 patients. J Am Acad Dermatol. 2015;73:76-82.

56. Hui-Lan Y, Xiao-Yan H, Jian-Yong F, Zong-Rong L. Combination of 308-nm excimer laser with topical pimecrolimus for the treatment of childhood vitiligo. Pediatr Dermatol. 2009;26:354-6.

57. Saraceno R, Nistico SP, Capriotti E, Chimenti S. Monochromatic excimer light $308 \mathrm{~nm}$ in monotherapy and combined with topical khellin $4 \%$ in the treatment of vitiligo: A controlled study. Dermatol Ther. 2009;22:391-4.

58. Ostovari N, Passeron T, Zakaria W, Fontas E, Larouy JC, Blot JF. Treatment of vitiligo by 308-nm excimer laser: An evaluation of variables affecting treatment response. Lasers Surg Med. 2004;35:152-6.

59. Bae JM, Jung HM, Hong BY, Lee JH, Choi WJ, Lee $\mathrm{JH}$, et al. Phototherapy for Vitiligo: A systematic review and meta-analysis. JAMA Dermatol. 2017;153:666-74.

6o. Calzavara-Pinton PG, Ortel B, Carlino AM, Honigsmann H, De Panfilis G. Phototesting and phototoxic side effects in bath PUVA. J Am Acad Dermatol. 1993;28:657-9.

61. Koulu LM, Jansén CT. Skin phototoxicity variations during repeated bath PUVA exposures to 8-methoxypsoralen and trimethylpsoralen. Clin Exp Dermatol. 1984;9:64-9.

62. Valkova S, Trashlieva M, Christova P. Treatment of vitiligo with local khellin and UVA: comparison with systemic PUVA. Clin Exp Dermatol. 2004;29:180-4.

63. Carlie G, Ntusi NB, Hulley PA, Kidson SH. KUVA (khellin plus ultraviolet A) stimulates proliferation and melanogenesis in normal human melanocytes and melanoma cells in vitro. Br J Dermatol. 2003;149:707-17.

64. Camacho F, Mazuecos J. Oral and topical L-phenylalanine, clobetasol propionate, and UVA/sunlight--a new study for the treatment of vitiligo. J Drugs Dermatol. 2002;1:127-31.

65. Camacho F, Mazuecos J. Treatment of vitiligo with oral and topical phenylalanine: 6 years of experience. Arch Dermatol. 1999;135:216-7.

66. Matin R. Vitiligo in adults and children: Surgical interventions. BMJ Clin Evid. 2015:20;2015.

67. Mulekar SV, Isedeh P. Surgical interventions for vitiligo: An evidence-based review. Br J Dermatol. 2013;169:57-66.

68. Lahiri K. Evolution and evaluation of autologous mini punch grafting in vitiligo. Indian J Dermatol. 2009;54:159-67.

69. Dillon AB, Sideris A, Hadi A, Elbuluk N. Advances in vitiligo: An update on medical and surgical treatments. J Clin Aesthet Dermatol. 2017;10:15-28.

70. Falabella R. The minigrafting test for vitiligo: Validation of a predicting tool. J J Am Acad Dermatol. 2004;51:672-3.

71. Falabella R. Treatment of localized vitiligo by autologous minigrafting. Arch Dermatol. 1988;124:1649-55. 